\title{
AOR
}

Selected Papers of \#AolR2020:

The 22nd Annual Conference of the

Association of Internet Researchers

Virtual Event / 13-16 Oct 2021

\section{CONSTRUCTING CONTEMPORARY PARENTHOOD IN DIGITAL SPACES: A WALKTHROUGH OF INFANT FEEDING APPLICATIONS}

\author{
Katrin Langton \\ Queensland University of Technology \\ Introduction
}

Infant feeding apps (IF apps) are mobile applications designed to facilitate the feeding and care of children in their first year of life (i.e. infancy). They commonly include 'babytracking' features that promote the recording and tracking of basic caring activities like feeding, nappy changes and sleep, with some including informational support, like breastfeeding education (Dienelt et al., 2019). Infant feeding apps continue to be extremely popular, with apps like Feed Baby being downloaded millions of times (Feed Baby, n.d.), making them important mediators of how early parenthood is experienced and practiced.

Previous studies have considered infant feeding or baby-tracking apps as part of a wider ecology of mobile applications designed to manage reproductive health, such as period trackers, fertility and pregnancy apps (Lupton, 2017).

Research on IF apps to-date has explored how care and surveillance become entangled in digitally mediated tracking-as-care regimes that surveil and discipline women's bodies specifically (Thornham, 2019); these issues also relate to broader trends around the datafication of family life (Barassi, 2020), which can produce valuable data-streams that contribute to surveillance capitalism (Zuboff, 2015).

\section{What this study does}

Similar to Livingstone's recent critique of media research approaches to datafication (2019), this study contends that a persistent focus on critical perspectives on parenting apps, and their (more or less perceived) power to discipline users into contributing to their own surveillance and datafication, disproportionately constructs users as disempowered, and lacking agency. Yet, aspects of app design that may support "resistance, reappropriation and disruption" (Thornham, 2019), remain underresearched.

This paper is a work-in-progress that explores how contemporary parenthood is constructed and mediated through the functionalities and technological design of infant

Suggested Citation (APA): Langton, K. (2021, October). Constructing Contemporary Parenthood in Digital Spaces: Walkthrough of Infant Feeding Applications. Paper (or panel) presented at AolR 2021: The 22nd Annual Conference of the Association of Internet Researchers. Virtual Event: AolR. Retrieved from http://spir.aoir.org. 
feeding apps. It aims to strike a balance between critical feminist analysis and the identification of opportunities for user resistance, agency and empowerment.

\section{Methodology}

The app walkthrough method (Light et al., 2018) was employed to facilitate a close examination of Australian IF apps Feed Baby and mum2mum. This method incorporates an exploration of an app's environment of expected use, encourages the researcher to mimic everyday use while remaining aware of elements of the interface that shape the flow and experience of app use, and examines digital traces of users (e.g. user reviews) to shed light on unexpected uses. It thereby provides insights into developers' agendas and socio-cultural influences that become embedded in technological design, and provides clues on the user experience.

\section{Initial Findings}

As its name suggests, mum2mum, published by the Australian Breastfeeding Association (ABA), specifically targets mothers as users. Similar to the international La Leche League, the ABA's organisational goals are to promote peer-to-peer support and breastfeeding education, to increase women's self-efficacy in breastfeeding, and their agency in health decision making, thereby countering the medicalisation of infant feeding (Jamieson, 2012); these goals are evident in mum2mum's design.

Recording functions (feeds, nappies, sleep) are provided, but their use is discouraged, and visual and discursive elements of the app foreground the importance of the motherinfant-bond, through the inclusion of highly affective visual depictions of infant feeding. In an online community of like-minded mothers, these images can indicate a mutual recognition and acknowledgement of the specificity of women's embodied experience of motherhood (Van Cleaf, 2020). Yet, these framings also naturalise women into the primary caregiving role based on their biology, and the app's informational material promotes a baby-led parenting philosophy that may alienate mothers who do not relate to this self-sacrificial maternal subjectivity.

Compared to other IF apps however, mum2mum may fill a niche for mothers who align with a mothering philosophy that promotes a re-valuation of women's embodied experience over the reduction of relational practices, like infant feeding, to numerical values.

In contrast to mum2mum's focus on embodiment, Feed Baby provides tools to "track every aspect of your newborn baby" (Feed Baby, n.d.), by enabling the daily recording of infant caring practices, and their tracking over time, without providing other instructive parenting support. Tracking is enabled through a reduction of infant caring activities to quantities and durations.

These reductions are common in IF apps, and support the kinds of tracking-as-care regimes criticised by Thornham (2019), that can contribute to the surveillance and disciplining of mothers in perinatal public health contexts (Lee, 2018). Third party access (e.g. health professionals, advertisers) to the information recorded in IF apps 
can contribute to the normalisation of the unnecessary surveillance and disciplining of female bodies. However, Feed Baby collects minimal personal information and allows parents control over who to share recorded data with, providing opportunities for empowerment. Parents' own experiential knowledge of the context of data collection, together with the ability to control access to records of babies' routines, can facilitate power-sharing in interactions with health-professionals (Stern, 2012), as well as making parents feel more in control during a period of their lives that is often experienced as overwhelming (Lupton, 2017).

\section{Conclusion}

The findings highlighted above indicate that infant feeding apps can be very diverse in design, warranting exploration as a distinct category of tools within the range of reproductive health apps available.

Even the heavily-gendered mum2mum provides opportunities for empowerment, through its maternalist approach to infant feeding and care, by providing an alternative to IF apps focussed on quantification.

Feed Baby, despite its focus on quantification and tracking, also holds empowering potential, by enabling a greater sense of control throughout a chaotic life-stage, as long as parents can remain the gatekeepers of the recorded information.

However, similar to the feminist critiques mentioned earlier, these findings are rather techno-deterministic; a deeper exploration of users' perspectives (e.g. inclusion of user interviews), is necessary to provide more meaningful insights into different contexts of app use, and how it mediates parents' lived experience and practice of contemporary parenthood.

\section{References}

Barassi, V. (2020). Datafied times: Surveillance capitalism, data technologies and the social construction of time in family life. New Media \& Society, 22(9), 1545-1560. https://doi-org.ezp01.library.qut.edu.au/10.1177\%2F1461444820913573

Dienelt, K., Moores, C., Miller, J., \& Mehta, K. (2019). An investigation into the use of infant feeding tracker apps by breastfeeding mothers. Health Informatics Journal. https://doi-org.ezp01.library.qut.edu.au/10.1177\%2F1460458219888402

Feed Baby. [@FeedBaby]. (n.d.). Feed Baby [Facebook site]. Facebook. https://www.facebook.com/FeedBaby

Jamieson, G. G. (2012). Working Together for Health. In Reaching for Health: The Australian women's health movement and public policy (pp. 155-178). ANU Press.

Lee, R. (2018). Biopower, Medicalization, and Maternalism. In The Ethics and Politics of Breastfeeding (pp. 33-67). University of Toronto Press. 
Light, B., Burgess, J., \& Duguay, S. (2018). The walkthrough method: An approach to the study of apps. New Media \& Society, 20(3), 881-900. https://doi-

org.ezp01.library.qut.edu.au/10.1177\%2F1461444816675438

Livingstone, S. (2019). Audiences in an Age of Datafication: Critical Questions for Media Research. Television \& New Media, 20(2), 170-183.

https://doi.org/10.1177\%2F1527476418811118

Lupton, D. (2017). 'It Just Gives Me a Bit of Peace of Mind': Australian Women's Use of Digital Media for Pregnancy and Early Motherhood. Societies, 7(3), 1-13.

https://doi.org/10.3390/soc7030025

Stern, M., Cotton, S., \& Drentea, P. (2012). The Separate Spheres of Online Health. Journal of Family Issues, 33, 1324-1350. https://doi.org/10.1177\%2F0192513X11425459

Thornham, H. (2019). Algorithmic vulnerabilities and the datalogical: Early motherhood and tracking-as-care regimes. Convergence, 25(2), 171-185.

https://doi.org/10.1177\%2F1354856519835772

Van Cleaf, K. M. (2020). The Pleasure of Connectivity: Media, Motherhood, and the Digital Maternal Gaze. Communication, Culture \& Critique, 1-18.

https://doi.org/10.1093/ccc/tcz045 